\title{
La responsabilité sociale des institutions de formations en santé
}

\author{
Social accountability of health education institutions
}

La responsabilité sociale (RS) devient un enjeu contemporain majeur dans nos sociétés où la précarité est préoccupante, les injustices évidentes, la mixité sociale une réalité, la mobilité complexe, les réseaux sociaux un moyen puissant de communication, l'impact des changements climatiques sur la santé encore largement inconnu. Elle concerne certes les facultés de médecine et les institutions de formation des professionnels de la santé, mais aussi l'ensemble de la société. Pour les premières, la prise de conscience date des années 1990 et le mouvement s'est amplifié ces dernières années au point de devenir un mouvement mondial. Dans le milieu francophone, depuis 2006, la Société internationale francophone d'éducation médicale (SIFEM), suivie par la Conférence internationale des doyens et des facultés de médecine d'expression française (CIDMEF) ont pris la mesure de ces changements. Aujourd'hui 56 facultés de médecine francophones dans 18 pays se sont engagées dans le projet francophone de recherche-action sur la RS. Par ailleurs, des publications de plus en plus nombreuses attestent le développement de la RS et son importance.

C'est donc le moment de faire le point sur ce mouvement et d'en décrire les concepts, les enjeux, les réalisations et les promesses pour le futur. Le dossier qui suit propose plusieurs contributions successives, qui ont été élaborées à partir des présentations effectuées lors de la session plénière qui était consacrée au thème de la RS, lors du congrès de la SIFEM à Bruxelles en avril 2015.

Une analyse s'impose d'abord à distance de ce qui se vit dans les facultés, pour interroger l'idée de la $\mathrm{RS}$, poser un regard critique, questionner la $\mathrm{RS}$ dans une perspective historique ou encore le caractère novateur du concept appliqué aux facultés de médecine. En envisageant le lien entre médecine et société, et en examinant la place de celle-ci dans la formation médicale. Céline Lefève, philosophe, nous invite à réfléchir aux moyens de mettre en place les 10 axes du consensus mondial. Elle envisage le rôle complémentaire des sciences sociales, l'élargissement de l'éthique aux problématiques sociales, intégrant une dimension individuelle autant que collective.

Charles Boelen, auteur des premières publications sur le sujet dans les années 90, mesure le chemin parcouru, rappelle l'historique et l'importance du concept, souligne les défis majeurs que pose l'application des axes du consensus mondial. Il nous rappelle que l'application de la RS concerne l'ensemble des activités de la faculté, aussi bien dans le domaine de l'enseignement que dans celui de la recherche ou du service à la communauté. Il insiste pour que l'on prenne la mesure de ce que la RS change pour le futur des praticiens car la faculté est concernée de manière importante par le devenir de ses diplômés, le choix de leur spécialité et leur lieu d'implantation, pour répondre aux besoins des patients. Il rappelle les valeurs et questions sousjacentes aux concepts. Enfin, il nous incite à considérer l'importance de la mesure de l'impact et de la qualité des réalisations, notamment dans une perspective d'accréditation qui s'imposera pour toutes les facultés d'ici 2023 pour la mobilité des professionnels de santé. Cette accréditation exigera une 
adaptation des normes utilisées comprenant des indicateurs de mesure des différentes activités de la faculté. Il souligne enfin les signes de mobilisation encourageants dans le monde francophone et appelle à un engagement individuel responsable.

Joel Ladner et ses collègues explicitent la démarche du comité de pilotage du projet francophone de recherche-action, comprenant l'ensemble des facultés participant au projet. Il décrit les résultats de la première phase de recherche-action et montre combien les acteurs du monde académique et de la société ont besoin de s'approprier les concepts qui sous-tendent la démarche de RS. Celle-ci est d'abord fonction des personnes et de leur degré de conviction, particulièrement celle du doyen, acteur clef pour développer activement la RS dans les facultés. Si tous conviennent de la nécessité d'obtenir un engagement sociétal fort, bien peu savent le décliner de manière explicite et adaptée au contexte local. Les enseignements tirés de la première phase de la recherche-action sur la RS dans le monde francophone sont utiles pour définir les stratégies futures du projet. Les nombreux étudiants qui ont participé à l'enquête ont montré un intérêt important pour la RS et cette démarche a apporté des réponses encourageantes pour le futur. Les exemples documentés dans l'article sont à la fois très riches et illustratifs de l'absolue nécessité de tenir compte des spécificités locales tout en intégrant des notions d'impact, de mesure, de partenariat large avec les autres professions médicales et les acteurs de la société. Les exemples décrits à Tours en France, en Tunisie, à Haïti ou à Madagascar nous montrent que les actions réalisées ont modifié en profondeur à la fois la perception des personnes concernées et les modalités pratiques de réalisation des objectifs d'apprentissage en vue de compétences élargies et adaptées.

Paul Grand'Maison et ses collègues présentent le plan stratégique mis en place pour les cinq prochaines années (2015-2020). Il souligne l'importance des valeurs qui sous-tendent le projet, la vision et les missions définies par une large consultation des personnes et facultés concernées, les axes choisis qui sont communs pour l'ensemble des facultés. L'accréditation des facultés de médecine est sans aucun doute l'enjeu majeur des prochaines années, d'autant plus que les agences internationales reconnues ont modifié leurs normes pour les mettre en adéquation avec les principes de RS. La mesure de l'impact des réalisations des facultés de médecine sera essentielle dans ce contexte pour illustrer les changements apportés dans l'adaptation des actions d'enseignement, de recherche et de service à la communauté. Les partenariats entre les professionnels de santé de disciplines différentes, les autres institutions de soins ou d'enseignement, ainsi que la société civile au sens large, comprenant les patients, est un autre enjeu majeur, gage d'une prise en compte des attentes et réalités dans lesquelles s'insèrent les facultés. Diffuser nos actions, publier leurs résultats, former les collègues, assurer un leadership à un niveau international ne pourra se réaliser sans une organisation professionnelle et des moyens adaptés.

Geneviève Moineau, présidente de l'Association des facultés de médecine canadiennes (AFMC), nous décrit la manière dont le Canada a mis en place des moyens et outils de réalisation de l'accréditation de ses 17 facultés de médecine, incluant des normes strictes et spécifiques concernant la RS. Elle précise que des protocoles d'entente ont été signés avec les Etats-Unis et les organismes mondiaux les plus importants comme le Liaison Committee on Medical Education ( $L C M E$ ), les normes étant reconnues par la Fédération mondiale pour l'éducation médicale. Elle décrit l'évolution de celles-ci de manière précise, et montre qu'un tel processus d'adaptation des normes prend du temps et nécessite un large partenariat, y compris avec la société civile. L'exemple canadien est un modèle pour les autres pays qui devront de toute évidence s'engager dans un tel processus basé sur des caractéristiques locales des systèmes de santé et d'éducation médicale, tout en étant redevables vis-à-vis des organismes supranationaux.

La RS constitue assurément un changement de paradigme non seulement pour l'éducation en sciences de la santé, mais aussi pour la société dans son ensemble. Pour les facultés de médecine, la RS ne peut se réduire à la santé publique ou à la première ligne de soins car elle concerne l'ensemble des disciplines médicales et, partant, la gouvernance de celles-ci. Que l'on pense par exemple à l'un des objectifs du plan stratégique, qui concerne 
la création de partenariats avec les autres professionnels de la santé, les acteurs responsables de la santé dans nos sociétés et les patients, directement concernés. Si chacun conviendra que cette collaboration est une réalité sur le terrain pour les professionnels de la santé, comment est-elle enseignée ? Avec quels résultats? Quel impact ? Comment est-elle organisée pour définir le choix des spécialités et les lieux de pratique future ? Une contribution récente montre à la fois que l'approche multi-professionnelle peut s'enseigner avec des résultats positifs, mais que les changements de comportements dans la pratique demeurent encore insuffisants ${ }^{[1]}$. D' autres exemples seraient utiles pour illustrer le fait que ces changements nécessitent du temps, des choix stratégiques motivés et des moyens considérables. Dans un monde plus enclin à la recherche d'efficience et de transparence, cette nouvelle posture de l'institution de formation s'imposera à l'avenir. De plus, un cadre général de normes illustrant la responsabilité sociale sera nécessaire pour une réactualisation de l'évaluation et de l'accréditation ${ }^{[2]}$.

En 1910, Abraham Flexner a transformé l'éducation médicale en introduisant une dimension scientifique de base solide, qui a conduit à l'organisation générale des curriculums en deux cursus successifs, respectivement pré-clinique puis clinique. On sait moins qu'il espérait que la fonction du médecin serait «sociale et préventive plutôt qu'individuelle et curative » ${ }^{[3]}$. Il écrivait en 1925 que «la médecine scientifique en Amérique -jeune, vigoureuse et positiviste- est aujourd'hui malheureusement déficiente en bases culturelles et philosophiques ${ }^{[4]}$. Si la base de connaissances a augmenté de manière exponentielle, les soins se sont complexifiés et les patients sont devenus plus exigeants. Certains aspects de la pratique professionnelle, comprenant le travail collaboratif, la politique de santé, l'organisation des soins, l'éthique ou la communication professionnelle sont encore insuffisamment valorisés dans un système de santé, par ailleurs de plus en plus fragmenté. Les aspects sociaux, économiques et politiques des soins, de même que le devenir du professionnel de la santé devront être mieux pris en compte. Au final, la capacité de s'adapter et de changer devront faire partie de la réalité du professionnel de la santé pour répondre aux besoins de la société.

Dominique PESTIAUX Centre Académique de Médecine Générale Faculté de Médecine, Université Catholique de Louvain, Bruxelles, Belgique Mailto : dominique.pestiaux@mmransart.be

\section{Références}

1. Tsakitzidis G, Timmermans O, Callewaert N, Truijen S. Meulemans H, Van Royen P. Participant evaluation of an education module on interprofessional collaboration for students in healthcare studies. BMC Medical Education 2015;15:188. DOI 10.1186/ s12909-015-0477-0.

2. Boelen C, Grand'Maison P, Ladner J, Pestiaux D. Responsabilité sociale et accréditation. Une nouvelle frontière pour l'institution de formation. Pédagogie Médicale 2008;9:235-44.

3. Boelen C. A new paradigm for medical schools a century after Flexner's report. Bull World Health Organ 2002;80:592-3.

4. Cooke M, Irby D, Sullivan W, Ludmerer K. American Medical Education 100 Years after the Flexner Report. N Engl J Med 2006;355:1339-44. 